\title{
The representational and processing characteristics of scripts
}

\author{
FRANCIS S. BELLEZZA \\ Ohio University, Athens, Ohio 45701 \\ and \\ GORDON H. BOWER \\ Stanford University, Stanford, California 94305
}

\begin{abstract}
Memory schemata may influence the storage of information in memory either by enabling information relevant to the instantiated schema to be more easily comprehended or by selectively allocating processing resources to information that fits the schema. In the experiment reported here, decision times, recall performance, and recognition performance were either typical or atypical of the scripts in which they were embedded. It was found that atypical actions were processed for longer periods of time than were typical actions and that recall performance for the two types of actions was the same. However, recognition of the typical actions was inferior to recognition performance on atypical actions, probably because subjects had difficulty discriminating between those typical actions that were presented and those that were inferred. It was concluded that memory schemata may affect the storage of information both by providing an ideational scaffolding for new information relevant to the schema and by influencing the degree of processing that relevant vs. nonrelevant information receives.
\end{abstract}

The role of memory schemata in learning and comprehension and the nature of these schemata have become major issues in cognitive psychology (Anderson, 1979; Rumelhart, 1980; Thorndyke \& Hayes-Roth, 1979). The idea of a memory schema is not a new one in psychology (Bartlett, 1932; Piaget, 1926), but only recently has the notion become more widely accepted. Schemata can be thought of as data structures useful for representing generic concepts stored in memory (Rumelhart \& Ortony, 1977) or as a cluster of knowledge that describes the typical properties of the concept it represents (Thorndyke \& Yekovich, 1980). Memory schemata have several properties that provide them with explanatory power (Rumelhart, 1980). In this paper, we focus on two major ways in which a memory schema can influence what information is stored in memory.

When information is perceived that is similar to or appropriate to a particular schema in memory, that schema is activated, or "instantiated" (Anderson, 1979; Rumelhart \& Ortony, 1977; Schank \& Abelson, 1977). The instantiated schema can then influence interpretation of the incoming information and the amount of processing it receives. After instantiation, more of the

The authors would like to thank the Stanford Low-Overhead Time-Sharing System for making computer time available. This research was supported in part by Grant MH-13905 to Gordon H. Bower from the National Institute of Mental Health. Requests for reprints may be sent to either Francis S. Bellezza, Department of Psychology, Ohio University, Athens, Ohio 45701, or to Gordon H. Bower, Department of Psychology, Stanford University, Stanford, California 94305. processing that takes place is concept driven in a "topdown" fashion (Schank, 1973).

The instantiated memory schema influences what becomes stored in memory in two major ways (Anderson, 1979; Rumelhart, 1980; Bower, Note 1). One way is by providing a framework in memory for new information that matches the structure of the schema. Schema have "slots" to which new values of expected categories of information can be "bound" or stored (Rumelhart \& Ortony, 1977). For example, consider the restaurant script, a memory schema representing the stereotyped behavior of a diner in a restaurant. The slots of this schema allow the names of the food and drink mentioned in the episode to be stored. The restaurant schema also includes typical script actions, such as sitting down at a table, having a drink, ordering food, and so on. Because of the generic nature of a script with its slots for binding variables, information representing typical instances of the schema are more easily stored in memory than is information not related to the schema.

Theorists apparently agree that information consistent with an instantiated schema is more easily stored than is information that is not. Hence, considering only the representational properties of a schema, it is possible that text information that does not fit the schema may simply not be retained (Anderson, 1979; Bransford \& Johnson, 1973). Or this information may be stored in memory but given a special tag (Graesser, Gordon, \& Sawyer, 1979; Graesser, Woll, Kowalski, \& Smith, 1980). 
Somewhat surprisingly, a number of studies have found that presented information fitting the activated schema is recalled no better than is presented information not fitting the schema. Graesser et al. (1980) found that typical script actions were recalled more poorly than were atypical script actions when recall took place immediately after presentation. However, with a 1-week delay in testing, the typical information was recalled better. Also, Bower, Black, and Turner (1979) found that recall of the typical script actions was not significantly better than the recall of irrelevant information. To help explain these results, one characteristic of schema-based processing should be noted. Events not explicitly presented in a text may be inferred from the instantiated schema either during presentation or during testing. In reading a text based on the restaurant script, subjects may indicate that they remember reading that the diner looked at a menu, even though nothing was stated regarding a menu in the text. This inability to discriminate between presented and nonpresented schema-relevant information can adversely affect both the recall and the recognition of information presented that fits the schema (Bower et al., 1979; Graesser et al., $1979,1980)$. Studies using verbally based schemata that were created during the experimental session have also found that schema-related information is better recalled only if the subjects can discriminate among the various instantiations of the schema (Bower, 1974; Thorndyke \& Hayes-Roth, 1979; Bellezza \& Bower, Note 2).

A second way in which the instantiated schema influences what information is stored is by allocating attentional and other processing resources among the various kinds of information being processed (Anderson, 1978; Rumelhart, 1980; Bower, Note 1). The amount of processing a particular item of information receives can be measured by the amount of time it is processed in combination with the amount of concurrent processing of other information taking place (Norman \& Bobrow, 1975).

One reason that information not related to the instantiated schema is better recalled than expected is that a greater share of processing resources may be allocated to it than is allocated to schema-relevant information during processing (Bobrow \& Norman, 1975). Schank and Abelson (1977) indicate that scriptrelated information may be processed in less time than information not related to the instantiated schema. If recall is partly dependent on processing time, then recall of information not related to the schema could be better recalled than it otherwise would be. Anderson (1978) argues against this view, suggesting that extra attention is given to information relevant to the schema. Graesser et al. (1979, 1980) also argue against such differential resource allocation. They suggest that schema-related information receives neither more nor less attentional resources than does information not related to the schema. The poorer memory performance for presented information that fits the schema compared with information that does not fit the schema results only from the problem of discriminating between presented and inferred schema information during testing.

The primary purposes of the experiment described here were to determine if typical and atypical script actions in a text are processed for equal lengths of times and to determine how these times are related to recall performance. However, some other hypotheses were also tested. If nonscript information is specially tagged in some way (Graesser et al., 1979), then it might be expected that typical and atypical script activities would each tend to cluster together in recall. Also, the hypothesis that typical script actions presented in the text are more difficult to recognize later than are atypical script actions was tested. This should occur because the false alarm rate for unpresented typical script activities is high and not because the hit rate on presented typical script activities is low (Bower et al., 1979; Graesser et al., 1979, 1980). Subjects should make more recognition errors by accepting typical actions never presented than by rejecting previously presented typical actions.

\section{METHOD}

\section{Subjects}

Twenty undergraduates enrolled in the introductory psychology course at Stanford University volunteered to participate to fulfill a course requirement.

\section{Materials}

Eight scripts, each containing four actions typical of the script and four actions atypical or irrelevant to the script, were taken from the material developed by Bower et al. (1979). Each action was incorporated into a sentence either seven or eight words long.

\section{Procedure}

The title of each script and the eight sentences composing it were each presented on a CRT monitor attached to a NOVA computer. Typical and atypical script actions were presented alternately in each script, with half the scripts starting with a typical script action and half starting with an atypical script action. The subject had to press one of two buttons as quickly as possible after each sentence appeared. The button labeled "yes" indicated that the sentence belonged to the story being presented, and the button labeled "no" indicated that the sentence presented did not belong to the story. Half the subjects responded "yes" with their right hands and half with their left. When a story title appeared, it was surrounded by asterisks. The subject was instructed to read a title carefully and then to press the "yes" button. An example of a title used is "John Eats in a Restaurant." The eight sentences following this title were: "John bought a newspaper at the counter. He was seated at an empty table. He smoked his favorite brand of cigar. John looked at the very complete menu. He could not find the salt shaker. He sipped his ice water while waiting. John started to brush off his shoes. He put on his coat and gloves."

After presentation of all the stories, the eight script titles were presented in a random order and the subject was asked to write down the sentences that had been presented with each title. Next, a recognition test was administered. Two typical and two atypical actions presented in the story and two typical 
and two atypical actions not presented in the story composed the recognition test for each script. "Yes" and "no" decisions were made using a 6-point rating scale, with a rating of 6 meaning "positively yes" and a rating of 1 meaning "positively no." All tests of statistical significance were performed with alpha set to .01 .

\section{RESULTS}

A 2 by 4 analysis of variance was performed on the decision times, the proportion of "yes" responses during presentation, and the proportion of sentences recalled. The two factors were the typicality of the action in each story and the quarter of the story in which the action occurred, respectively. The results of the experiment are shown in Table 1. The mean decision time for typical script actions was significantly less than that for atypical actions $[\mathrm{F}(1,19)=40.00, \mathrm{MSe}=.846]$. As expected, the proportion of typical script actions judged as fitting into the story was significantly greater than the proportion of atypical script actions $[\mathrm{F}(1,19)=52.33, \mathrm{MSe}=.073]$. Also, the further into a story an action occurred, the less likely the sentence containing the actions was judged as fitting into the story. In each successive quarter of the story, the proportions of "yes" responses were $.79, .75$, .74 , and $.66[\mathrm{~F}(3,57)=11.50, \mathrm{MSe}=.011]$.

The sentences representing atypical script actions were recalled as well as were those representing typical script actions. Also, there was an overall primacy effect for recall. The proportions of sentences recalled from each quarter of the stories were $.65, .53, .48$, and .47 , respectively $[\mathrm{F}(1,57)=7.72, \mathrm{MSe}=.036]$.

When a second factor, "yes" vs. "no," was added to the analysis, no differences in recall level were found between typical and atypical script actions contingent on type of decision. Also, no relation was found between decision time and recall level.

The recognition results based on the 6-point rating scale showed that the subjects could discriminate between old and new items $[\mathrm{F}(1,19)=840.67, \mathrm{MSe}=$ $.682]$. Furthermore, old items were given higher recognition ratings both for atypical script actions and for typical script actions. However, the interaction between the newness of an item and its typicality was significant $[F(1,19)=94.24, \mathrm{MSe}=.136]$, with the discrimination performance for atypical items being superior. A Tukey HSD test (Kirk, 1968) showed that the mean rating of the old typical items was significantly lower than the

Table 1

Performance Characteristics of Typical and Atypical Script Actions

\begin{tabular}{lrc} 
& Typical & Atypical \\
\hline Decision Time (in Secs) & 3.72 & 4.64 \\
Proportion of "Yes" Decisions & .89 & .58 \\
Proportion of Sentences Recalled & .52 & .55 \\
Recognition Rating-Old & 5.21 & 5.66 \\
Recognition Rating-New & 1.99 & 1.31 \\
ARC Score & -.22 & \\
\hline
\end{tabular}

mean rating of the old atypical items. Also, the mean rating of the new typical items was significantly higher than the mean rating of the new atypical items.

Finally, no evidence was found for the clustering of typical and atypical actions in the recall sequences. The mean ARC score of -.22 (Roenker, Thompson, \& Brown, 1971) was not significantly different from zero. An ARC score of .0 means no clustering is occurring above the chance level, and an ARC score of 1.0 means that perfect clustering is taking place. Subjects tended to recall both types of sentences in the same order that they were presented.

\section{DISCUSSION}

The results show that atypical script actions were processed longer and were recalled as well as typical script actions were. Perhaps the simplest explanation for this result is that the typical script actions were more easily stored in memory, but the greater processing time for the atypical script actions improved their recall and made up for their storage deficit. Results similar to these were reported by Friedman (1979), who found that subjects spent more time looking at unexpected objects in naturalistic pictures. Both the structural features of an instantiated schema and the manner in which the schema influences the allocation of processing resources may affect later recall performance. It seems clear from the decision time data that differential resource allocation can indeed occur, although Graisser et al. (1979) have suggested otherwise.

In the present experiment, the proportion of typical and atypical actions presented in each script-based story were the same. However, if the proportion of atypical actions had been made small compared with the proportion of typical actions, a von Restorff effect (Waugh, 1969) might have occurred, with the recall of the atypical script items becoming superior to the recall of the typical items. The more unexpected the information with respect to the script, the greater the processing that may be allocated to it (Rundus, 1971).

The decrease in the proportion of "yes" responses with the increase in the serial position of both types of sentences suggests that the subjects were basing their decisions not only on generic script information, but also on some specific script information in memory. It is not yet clear how general this phenomenon is and under what conditions it may occur during script-based learning.

The recognition results for old and new atypical script actions correspond to those reported by Graesser et al. (1979, 1980). There was better discrimination for atypical than for typical old and new actions. However, the discrimination between old and new typical script actions found here was much better than that reported by Graesser et al. (1979). In the present experiment, subjects discriminated quite well between presented and nonpresented typical actions. This result does not support the "script-action-plus-tag" proposal of Graesser et al. (1979), which supposes that once a schema has been instantiated, all the information characteristic of that schema is also activated. That hypothesis implies that the subject cannot distinguish between those typical script actions that were presented and those that were not. This result was not obtained here, and it is unlikely to be generally. Probably, the recognition of all presented information depends on the amount of study time allotted to it. The presentation rate of sentences in this experiment was slower than that used by Graesser et al. (1979), which could account for the differing results. A somewhat more flexible model is needed for storing script-type information in memory than the "script-activation-plus-tagging" model. The theory proposed by Bower et al. (1979) can account for subjects' ability to discriminate between old and new typical 
script actions. However, that model predicted that presented typical script actions would receive higher recognition ratings compared with presented atypical actions, but the result obtained here was just the opposite.

The absence of clustering of typical and atypical script actions indicates that narrative actions are stored in memory and accessed according to their order of presentation. Currently, the notions of an unordered "weird list" (Schank \& Abelson, 1977) or of the tagging of nonscript information (Graesser et al., 1979) do not suffice to explain the proper ordering of recall.

The results of this experiment indicate that an instantiated memory schema determines what is stored in memory in a somewhat complex manner. The structural characteristics and "slots" of the schema insure that information that "fits" the schema is more easily stored than are other types of information. However, the schema may also play some role in determining the allocation of processing resources, so that information that does not "fit" may sometimes receive more processing. Just what memory mechanisms are involved in this extra processing are not clear, but they clearly enhance recall of the information. These results, as do other experimental results on memory schemata, emphasize the importance of prior knowledge in learning. However, they also demonstrate the subtle effects this knowledge may have on the processing and retention of new information.

\section{REFERENCE NOTES}

1. Bower, G. H. Review of recent research on schema and script learning. Paper presented at the Japan-USA Conference on Cognitive Science, Tokyo, September 1980.

2. Bellezza, F. S., \& Bower, G. H. A method for studying the formation of memory schemata. Paper presented at the annual meeting of the Psychonomic Society, St. Louis, Mo., November 1980.

\section{REFERENCES}

Anderson, R. C. Schema-directed processes in language comprehension. In A. Lesgold, J. Pellegrino, S. Fokkema, \& R. Glaser (Eds.), Cognitive psychology and instruction. New York: Plenum, 1979.

Bartuett, F. C. Remembering. Cambridge, England: Cambridge University Press, 1932.

Bobrow, D. G., \& Norman, D. A. Some principles of memory schemata. In D. G. Bobrow \& A. Collins (Eds.), Representation and understanding. New York: Academic Press, 1975.

BOWER, G. H. Selective facilitation and interference in retention of prose. Journal of Educational Psychology, 1974, 66, 1-8.
Bower, G. H., Black, J. B., \& TURner, T. J. Scripts in memory for text. Cognitive Psychology, 1979, 11, 177-220.

Bransford, J. D., \& Johnson, M. K. Considerations of some problems of comprehension. In W. G. Chase (Ed.), Visual information processing. New York: Academic Press, 1973.

Friedman, A. Framing pictures: The role of knowledge in automatized encoding and memory for gist. Journal of Experimental Psychology: General, 1979, 108, 316-355.

Graesser, A. C., Gordon, S. E., \& Sawyer, J. D. Recognition memory for typical and atypical actions in scripted activities: Tests of a script pointer tag hypothesis. Journal of Verbal Learning and Verbal Behavior, 1979, 18, 319-322.

Graesser, A. C., Woll, S. B., Kowalski, D. J., \& Smith, D. A. Memory for typical and atypical actions in scripted activities. Journal of Experimental Psychology: Human Learning and Memory, 1980, 6, 503-515.

KIRK, R. E. Experimental design procedures for the behavioral sciences. Belmont, Calif: Wadsworth, 1968.

Norman, D. A., \& Bobrow, D. G. On data-limited and resourcelimited processes. Cognitive Psychology, 1975, 7, 44-64.

Pinget, J. The language and thought of the child. New York: Harcourt, Brace, 1926.

Roenker, D. L., Thompson, C. P., \& Brown, S. C. Comparison of measures for the estimation of clustering in free recall. Psychological Bulletin, 1971, 76, 45-48.

Rumelhart, D. E. Schemata: The building blocks of cognition. In R. Spiro, B. Bruce, \& W. Brewer (Eds.), Theoretical issues in reading comprehension. Hillsdale, N.J: Erlbaum, 1980.

Rumelhart, D. E., \& Ortony, A. The representation of knowledge in memory. In R. C. Anderson, R. J. Spiro, \& W. E. Montague (Eds.), Schooling and the acquisition of knowledge. Hillsdale, N.J: Erlbaum, 1977.

Rundus, D. Analysis of rehearsal processes in free recall. Journal of Experimental Psychology, 1971, 89, 63-77.

Schank, R. C. Identification of conceptualizations underlying natural language. In R. C. Schank \& K. M. Colby (Eds.), Computer models of thought and language. San Francisco: Freeman, 1973.

Schank, R. C., \& Abelson, R. P. Scripts, plans, goals, and understanding. Hillsdale, N.J: Erlbaum, 1977.

Thorndyke, P. W., \& HAYes-Roth, B. The use of schemata in the acquisition and transfer of knowledge. Cognitive Psychology, 1979, 11, 82-106.

Thorndyke, P. W., \& Yekovich, F. R. A critique of schemabased theories of human story memory. Poetics, 1980, 9, 23-49.

WAUGh, N. V. C. Free recall of conspicuous items. Journal of Verbal Learning and Verbal Behavior, 1969, 8, 448-456.

(Received for publication May 13, 1981.) 